\title{
Formulation of Thermosensitive Hydrogel Containing Cyclodextrin for Controlled Drug Delivery of Camptothecin
}

\author{
Swatantra Kumar Singh Kushwaha ${ }^{1 *}$, Awani Kumar Rai ${ }^{1}$ and Satyawan Singh ${ }^{2}$ \\ ${ }^{1}$ Pranveer Singh Institute of Technology, Kanpur, ${ }^{2}$ Saroj Institute of Technology \& Management, Lucknow, India
}

${ }^{*}$ For correspondence: Email: swatantrakushwaha@yahoo.co.in; Tel: +91-9450560321

Received: 30 August 2013

Revised accepted: 1 May 2014

\begin{abstract}
Purpose: To formulate and evaluate temperature-sensitive, controlled-release camptothecin hydrogel for anticancer drug delivery.

Method: Temperature-sensitive hydrogel based on chitosan/ $\beta$-glycerophosphate $(\beta-G P) / \beta$-cyclodextrin $(\beta-C D)$ was prepared by crosslinking method. The formulations were characterized by Fourier transform infrared spectroscopy (FTIR), x-ray diffraction (XRD), gelation time, and viscometry, as well as for controlled release. The formulation, containing camptothecin, was studied by MTT assay on tumor cell MCF-7. The effectiveness of treatment was measured in terms of controlled tumor growth inhibition (TGI).

Results: The hydrogel formulation showed good properties in terms of $\mathrm{pH}$, gelation, viscosity and invitro release. The gelation temperature and viscosity of the formulation was optimum. Camptothecin (CPT) released from the hydrogel (TF8) over $8 \mathrm{~h}$ in $\mathrm{pH} 7.4$ buffer ranged from $38.97-92.5 \%$, and varied according to the composition of the hydrogels. Release of camptothecin was lowest from preparations without cyclodextrins. Tumor growth inhibition activity of CPT in MCF-7 cell was highest for the formulation containing $1 \%$ chitosan, $8 \% \beta-G P$ and $1 \% \beta-C D$ while no inhibition was observed for the blank temperature sensitive hydrogel formulation.

Conclusion: These formulations are a promising and more effective delivery system that can be developed to serve as an alternative to the conventional system for anticancer drug delivery.
\end{abstract}

Keywords: Hydrogel, Chitosan, $\beta$-Glycerophosphate, $\beta$-Cyclodextrin, Camptothecin, MCF-7 cell line

Tropical Journal of Pharmaceutical Research is indexed by Science Citation Index (SciSearch), Scopus, International Pharmaceutical Abstract, Chemical Abstracts, Embase, Index Copernicus, EBSCO, African Index Medicus, JournalSeek, Journal Citation Reports/Science Edition, Directory of Open Access Journals (DOAJ), African Journal Online, Bioline International, Open-J-Gate and Pharmacy Abstracts

\section{INTRODUCTION}

20(S)-Camptothecin (CPT) is a cytotoxic quinoline alkaloid which binds to the complex of the enzyme topoisomerase-I with DNA during replication, and thereby stabilizing it. This prevents DNA re-ligation and therefore causes DNA damage which results in apoptosis. In this sense, CPT has shown significant antitumor activity in a broad spectrum of human malignancies [1]. CPT holds a pH-dependent equilibrium in aqueous medium between the lactone and the carboxylate forms. The former is essential for anticancer activity, whereas the carboxylate is almost inactive [2]. Unfortunately, the clinical application of CPT is hampered by its poor pharmaceutical profile, with extreme aqueous insolubility, low stability of the lactone form at physiological $\mathrm{pH}$, and severe systemic toxicities which included myelosuppression, vomiting, diarrhea, and hemorrhagic cystitis [3-5]. Cyclodextrins (CD) have lipophilic inner cavities and hydrophilic outer surfaces. They are capable of interacting with a large variety of guest molecules to form non-covalent inclusion complexes. Cyclodextrins are cyclic 
oligosaccharides and contain at least six D-(+) glucopyranose units which are attached by a- $(1$, 4) glucosidic bonds. They have been widely used for the formulation of drugs having bioavailability problems resulting from poor aqueous solubility, poor stability (hydrolytic or photodegradation, etc.) and severe side effects [6-8].

In the present work, we have used a chitosan polymer to formulate a biodegradable and biocompatible formulation for controlled delivery of camptothecin in a slow-release manner directly into a tumor cell. In this paper, we report the in vitro release characteristics of the camptothecin-polymer hydrogel and the in vitro effect of delivering camptothecin in different concentrations to a MFC7 tumor cell. The delivery vehicle used is one of a family of thermosensitive chitosan solutions, formulated at physiological $\mathrm{pH}$, which remain liquid at low temperatures and turn into gel when heated. The polymeric matrix used in this study consists of chitosan polymer and $\beta$-glycerophosphate $(\beta$ GP). Addition of glycerol-2-phosphate ( $\beta$-GP) to chitosan solution produces a hydrogel which undergoes sol-gel transition at a temperature close to $37{ }^{\circ} \mathrm{C}$, making the formulation a suitable vehicle for drug administration since the hydrogel when implanted into the body, flows to fill voids or cavities and becomes solid at body temperature. These hydrogels are suitable carriers for water-insoluble drugs and they are non-toxic and highly biocompatible. Chitosan is an important natural polymer widely used for medical and pharmaceutical applications.

\section{EXPERIMENTAL}

\section{Materials}

Chitosan (deacetylation degree, DDA $=80 \%$ ) was obtained from HiMedia Laboratories Pvt. Ltd., Mumbai, India. Chitosan flakes were dissolved in aqueous hydrochloric acid $(0.1 \mathrm{~N})$, filtered, dialyzed and precipitated with aqueous $\mathrm{NaOH}(6 \mathrm{~N})$. The precipitated chitosan was washed several times with water and vacuum dried. The white chitosan powder obtained was stored in a closed flask until used. Camptothecin (CPT) obtained from Coral Drugs, New Delhi and $\beta$-glycerophosphate $(\beta-G P)$ and $\beta$-cyclodextrin $(\beta-C D)$ were obtained from HiMedia Laboratories Pvt. Ltd., Mumbai, India. Demineralized and double distilled water was used. All chemicals and reagents used were of analytical grade.

\section{Preparation of autogelling chitosan solution}

Chitosan solutions were prepared in $0.1 \mathrm{~m}$ hydrochloric acid at room temperature. The chitosan powders were progressively added to the solvent with stirring and mixture was stirred for a further $3 \mathrm{~h}$. Sterile formulations were obtained by autoclaving $\left(121{ }^{\circ} \mathrm{C}, 20 \mathrm{~min}\right)$ [9]. Chilled $\beta$-GP aqueous solution (sterilized through a $0.22 \mu \mathrm{m}$ filter) was carefully added to $18 \mathrm{ml}$ of cooled chitosan solution drop wise to obtain clear and homogeneous liquid solution in a final volume of $20 \mathrm{ml}$. This ratio of Chitosan: $\beta-G P$ had a thermogelling temperature of $37^{\circ} \mathrm{C}$. The final solutions were mixed for an additional $10 \mathrm{~min}$ at $4{ }^{\circ} \mathrm{C}$. The $\mathrm{pH}$ of the final cold solutions was 6.9.

\section{Preparation of chitosan/cyclodextrin solution}

Homogeneous clear chitosan/hydrochloric acid solutions were prepared then added to $\beta-C D$ at room temperature by homogenously dispersing the powered $\beta-C D$ in chitosan solution under aseptic condition.

\section{Preparation of chitosan/ $\beta-$ GP/ $\beta-C D$ loaded with camptothecin}

Chitosan/ $\beta-G P / C D / C P T$ formulations were prepared at room temperature by homogeneously dispersing the powdered camptothecin in chitosan solutions under aseptic conditions. The $\beta$-GP solution was added slowly to the cooled camptothecin/chitosan dispersion under aseptic conditions.

Table 1: Composition of temperature-sensitive hydrogel

\begin{tabular}{llllll}
\hline $\begin{array}{l}\text { Formulation } \\
\text { code }\end{array}$ & $\begin{array}{l}\text { CPT } \\
(\% \text { w/v) }\end{array}$ & $\begin{array}{l}\text { Chitosan } \\
(\% \mathbf{w} / \mathbf{v})\end{array}$ & $\boldsymbol{\beta}-\mathbf{G P}(\% \mathbf{w} / \mathbf{v})$ & $\begin{array}{l}\boldsymbol{\beta}-\mathbf{C D} \\
(\% \mathbf{w} / \mathbf{v})\end{array}$ & $\begin{array}{l}\mathbf{H C l} \\
(\mathbf{M})\end{array}$ \\
\hline TF & 0.5 & - & - & - & 0.1 \\
TF2 & 0.5 & 1.0 & 7.0 & 1.0 & 0.1 \\
TF3 & 0.5 & 1.0 & 7.0 & 1.5 & 0.1 \\
TF5 & 0.5 & 1.5 & 7.0 & 1.0 & 0.1 \\
TF6 & 0.5 & 1.5 & 7.0 & 1.5 & 0.1 \\
TF8 & 0.5 & 1.0 & 8.0 & 1.0 & 0.1 \\
TF9 & 0.5 & 1.0 & 8.0 & 1.5 & 0.1 \\
TF11 & 0.5 & 1.5 & 8.0 & 1.0 & 0.1 \\
TF12 & 0.5 & 1.5 & 8.0 & 1.5 & 0.1 \\
\hline
\end{tabular}




\section{Detection of CPT by HPLC}

Quantitative analysis was performed on a Shimadzu LC 2010C HT HPLC chromatographic system equipped with an Auto sampler, a solvent module, Detector and a System HP ChemStations system. The column was a reverse-phase RP18 column. The HPLC system was eluted isocratically with methanol: water $(63: 37 ; \mathrm{v} / \mathrm{v})$ at room temperature. The flow rate of the mobile phase was $1.0 \mathrm{ml} / \mathrm{min}$ and samples were measured at a wavelength of $370 \mathrm{~nm}$. A standard curve was constructed by plotting peak area against concentration. The assay was found to be highly accurate and reproducible.

\section{Fourier transform infrared spectroscopy (FTIR)}

It was performed, using a Perkin Elmer Spectrum Two spectrophotometer, to understand if there exists some interaction between drug and excipients. The spectra were obtained in the region from $4000 \mathrm{~cm}^{-1}$ to $650 \mathrm{~cm}^{-1}$.

\section{X-ray diffractometry}

The crystal X-ray scattering measurements for the obtained sample of camptothecin and its formulation were performed to determine the solid structure of drug. XRD patterns were obtained with a Seifert Germany ISO debyeflex 2002 apparatus (Japan) using $\mathrm{Cu}-\mathrm{K}$ a radiation $\left(\lambda=1.541841 A^{*}\right)$, a voltage of $40 \mathrm{kV}$ and a 100 $\mathrm{mA}$ current. Samples were scanned from 0-600 $2 \theta$ for qualitative studies and the scanning rate was $40 / \mathrm{min}$.

\section{In vitro gelation and viscosity studies}

The two main prerequisites of an in situ gelling system are viscosity and gelling capacity (speed and extent of gelation). The formulation should have an optimum viscosity that will allow easy injectable into the body as a liquid (drops), which would undergo a rapid sol-to-gel transition. Additionally, to facilitate sustained release of drug to the tumoral tissue, the gel formed in situ should preserve its integrity without dissolving or eroding for a prolonged period of time. Viscosity of injected formulation is an important factor in determining residence time of drug in the injected area. The developed formulations were poured into the small sample adaptor of the Brookfield DV-II + Pro viscometer, RV spindle 6 and the angular velocity increased gradually from 0.5 to $50 \mathrm{rpm}$. The hierarchy of the angular velocity was reversed. The average of the three readings was used to calculate the viscosity.

\section{Standard calibration curve for CPT}

Accurately weighed $10 \mathrm{mg}$ camptothecin was dissolved in $100 \mathrm{ml}$ of phosphate buffer $\mathrm{pH} 7.4$ to get the stock solution of $100 \mu \mathrm{g} / \mathrm{ml}$. From this stock solution, aliquots of $1 \mathrm{ml}$ was withdrawn and get the stock solution of $10 \mu \mathrm{g} / \mathrm{ml}$. From this stock solution, aliquots of $1,2,3,4,5$ and $6 \mathrm{ml}$ were withdrawn and further diluted to $10 \mathrm{ml}$ with buffer to obtain a concentrations range of $1,2,3$, 4,5 and $6 \mu \mathrm{g} / \mathrm{ml}$. The absorbance of the solutions was measured at $285 \mathrm{~nm}$ by using UVVis spectrophotometer.

\section{In vitro release studies}

The release profile of a drug predicts how a delivery system might function and gives valuable insight into its in vivo behavior. All the temperature sensitive and $\mathrm{pH}$ sensitive in situ gelling formulations of CPT were subjected to in vitro release studies. These in vitro release studies were carried out using potassium phosphate buffer of $\mathrm{pH} 7.4$ as the dissolution medium.

Approx 1.2 inch length of the dialysis tube was taken and then soaked overnight in the phosphate buffer $7.4 \mathrm{pH}$. Now the amount of CPT equivalent to $10 \mathrm{mg}$ of drug was calculated and placed in the dialysis tube whose ends were tied with a thread to prevent leakage. The dialysis tube bags were then placed in $100 \mathrm{ml}$ of phosphate buffer $7.4 \mathrm{pH}$ placed in the shaking water bath and maintained at $37{ }^{\circ} \mathrm{C}$ with a frequency of 50 shakings per minute. Aliquots of $2 \mathrm{ml}$ were withdrawn and filtered and sink condition maintained using phosphate buffer The filtrate obtained was then suitably diluted 10 times $(1 \mathrm{ml}$ filtrate up to $10 \mathrm{ml}$ ) and the absorbance taken after scanning. The experiment was carried out in triplicate. Drug release over $8 \mathrm{~h}$ is shown in Table 3 . The amount of drug loaded initially in the polymer was determined by extracting with methanol to release the residual camptothecin.

\section{In vitro cytotoxicity studies}

The in vitro cytotoxicity of the CPT formulations was performed on the human breast cancer cell line MCF-7. The concentration of drug was 10 $\mu \mathrm{g} / \mathrm{ml}$ used for in vitro studies. Sensitivity of MCF-7 cells to formulations was determined individually by the MTT colorimetric assay. Cells were seeded in a flat-bottomed 96-well plate and incubated for $24 \mathrm{~h}$ at $37{ }^{\circ} \mathrm{C}$ and in $5 \% \mathrm{CO}_{2}$. The cell line was exposed to all formulations mentioned above. The solvent DMSO treated 
cells served as control. Cells were then treated with MTT reagent $\left(20 \mu \mathrm{l} /\right.$ well) for $4 \mathrm{~h}$ at $37^{\circ} \mathrm{C}$ and then DMSO $(200 \mu \mathrm{l})$ was added to each well to dissolve the formazan crystals. The optical density (OD) was recorded at $492 \mathrm{~nm}$ in a microplate reader. Percentage of residual cell viability was determined as in Eq 1.

$\mathrm{RCV}(\%)=[1-(\mathrm{ODt} / \mathrm{ODc})] \times 100$

where RCV is the residual cell viability, ODt is the OD of treated cells and ODc is the OD of control cells.

\section{RESULTS}

\section{Fourier transform infrared spectroscopy (FTIR) spectra}

The FTIR spectra of drug CPT, Chitosan, $\beta-G P$, $\beta-C D$ and physical mixture of CPT are shown in Figure 1. The FTIR studies showed that there no interactions between CPT and excipients. The main characteristic peaks of CPT are at around $1750,1460-1600,1270-1290 \mathrm{~cm}^{-1}$. The FTIR spectrum of physical mixture of CPT showed their peaks according to drug.

\section{In vitro gelation and viscosity studies}

The gelation temperature and the viscosity of the formulated hydrogel are shown in Table 2. The gelation temperature of the hydrogel formulation was in range of 34.0 to $37.3^{\circ} \mathrm{C}$ and the viscosity of the hydrogels was in range of $2522.1 \mathrm{cP}$ to $6213.5 \mathrm{cP}$ at $20 \mathrm{rpm}$ and $25^{\circ} \mathrm{C}$.

\section{In vitro cytotoxicity}

In vitro cytotoxicity data are shown in Figure 2. The hydrogel containing $0.5 \% \quad(\mathrm{w} / \mathrm{v})$ camptothecin was more effective than the one without hydrogel in terms of growth inhibition. Tumors injected with blank chitosan/ $\beta-G P / \beta-C D$ showed no inhibition of growth of tumor, confirming that the hydrogel alone has no effect on tumor growth.

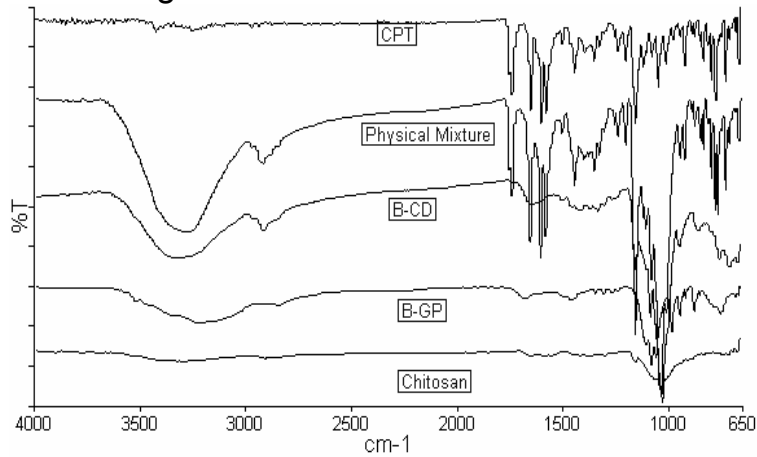

Figure 1: FTIR spectra of drug and excipients

Table 2: Gelation time and viscosity of temperature sensitive formulations

\begin{tabular}{lccc}
\hline $\begin{array}{l}\text { Formulation } \\
\text { code }\end{array}$ & $\mathbf{p H}$ & $\begin{array}{c}\text { Gelation } \\
\text { Temperature } \\
\left({ }^{\circ} \mathbf{C}\right)\end{array}$ & $\begin{array}{c}\text { Viscosity (cP) } \\
\left(\mathbf{2 0} \mathbf{~ r p m , ~ 2 5} \mathbf{~}^{\circ} \mathbf{C}\right)\end{array}$ \\
\hline TF & 7.20 & $35.3 \pm 1.31$ & $2522.1 \pm 12.38$ \\
TF2 & 7.22 & $36.6 \pm 0.45$ & $2929.2 \pm 13.07$ \\
TF3 & 7.11 & $36.2 \pm 1.33$ & $3836.9 \pm 16.92$ \\
TF5 & 6.99 & $34.0 \pm 2.03$ & $4313.1 \pm 15.43$ \\
TF6 & 7.00 & $35.9 \pm 1.93$ & $4938.2 \pm 13.56$ \\
TF8 & 7.43 & $36.4 \pm 1.63$ & $3248.6 \pm 19.67$ \\
TF9 & 7.33 & $37.3 \pm 0.09$ & $3989.0 \pm 24.11$ \\
TF11 & 6.98 & $35.7 \pm 0.19$ & $5003.0 \pm 22.79$ \\
TF12 & 7.00 & $36.5 \pm 0.23$ & $6213.5 \pm 26.46$ \\
\hline \multicolumn{4}{l}{ The gelation time \& viscosity data is (Mean $\pm S D, n=3)$} \\
for temperature sensitive hydrogel
\end{tabular}

18.56

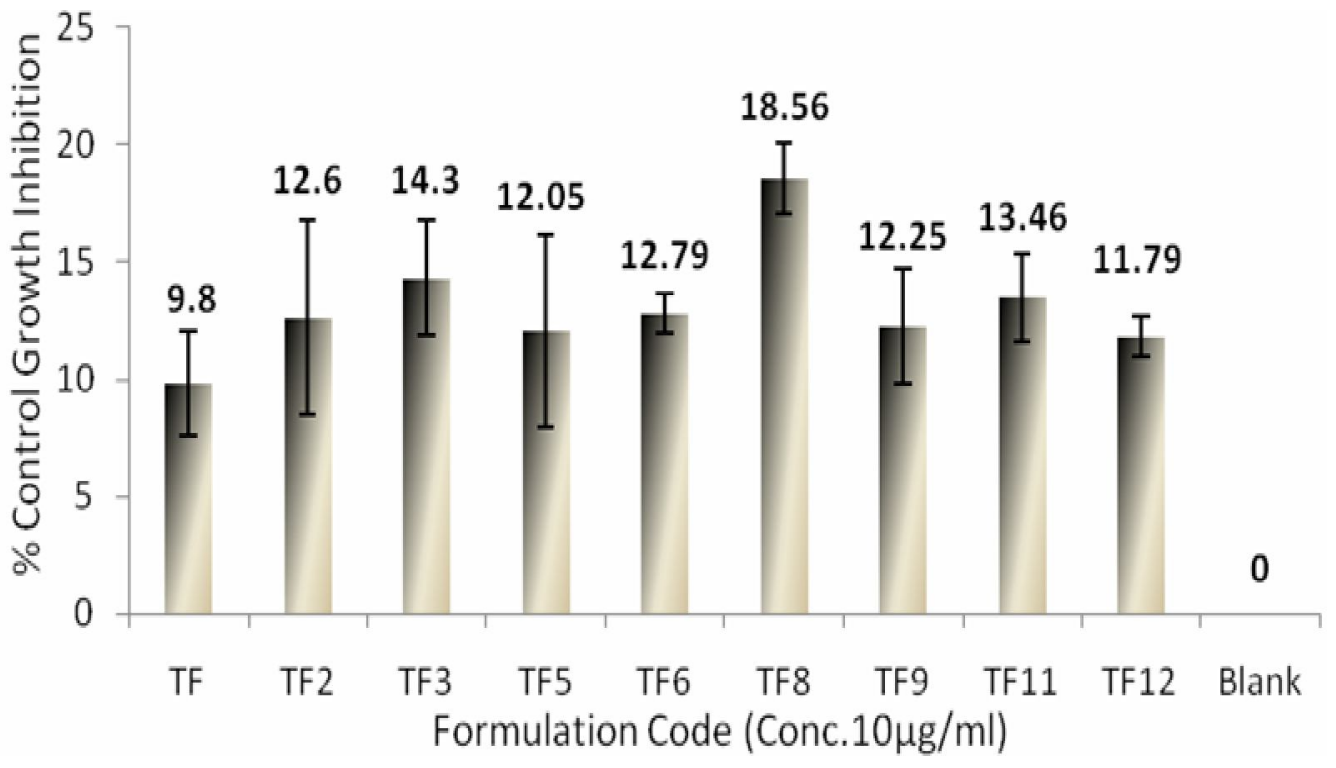

Figure 2: In vitro study on MCF-7 Tumor Cell line, \% control growth inhibition data (Mean $\pm S D, n=3$ ) for temperature sensitive hydrogel formulations 
Table 3: The $\%$ cumulative drug release from different formulation

\begin{tabular}{|c|c|c|c|c|c|c|c|c|c|}
\hline $\mathrm{Hr}$. & TF & TF2 & TF3 & TF5 & TF6 & TF8 & TF9 & TF11 & TF12 \\
\hline 0.5 & $00.25 \pm 0.21$ & $11.66 \pm 2.22$ & \pm 2.12 & $10.51 \pm 0.24$ & $09.23 \pm 1.82$ & $23.33 \pm 1.16$ & $16.92 \pm 0.44$ & $8.33 \pm 0.08$ & $0.64 \pm 0.25$ \\
\hline 1.0 & $01.02 \pm 0.63$ & $16.79 \pm 2.33$ & $23.33 \pm 3.22$ & $14.35 \pm 0.68$ & $14.61 \pm 1.28$ & $27.17 \pm 3.25$ & $28.46 \pm 0.64$ & $31.28 \pm 4.44$ & $01.53 \pm 1.67$ \\
\hline 1.5 & $03.97 \pm 1.42$ & $20.38 \pm 2.31$ & $27.17 \pm 1.12$ & $21.15 \pm 2.50$ & $21.66 \pm 0.62$ & $33.97 \pm 1.67$ & $31.41 \pm 0.24$ & $33.97 \pm 4.84$ & $13.46 \pm 3.22$ \\
\hline 2.0 & $08.46 \pm 0.94$ & $26.28 \pm 2.98$ & $32.30 \pm 2.90$ & $23.46 \pm 1.4$ & $24.74 \pm 0.86$ & 36.28 & 35 & 2.32 & 1.82 \\
\hline 2.5 & $11.66 \pm 2.70$ & $32.43 \pm$ & $38.33 \pm 1.16$ & $32.69 \pm 0$ & 33.20 & 45.76 & 38 & 25 & 88 \\
\hline 3.0 & $12.94 \pm 0.52$ & $37.30 \pm 3.40$ & $40.89 \pm 3.25$ & $37.43 \pm 0.76$ & & $50.76 \pm 0.86$ & $39.51 \pm 1.98$ & $42.17 \pm 1.67$ & \\
\hline 3.5 & $16.92 \pm 0.68$ & $42.94 \pm 2.62$ & $48.46 \pm 1.67$ & $38.58 \pm 2.50$ & & & & $45.00 \pm 3.22$ & \\
\hline 4.0 & $19.48 \pm 0.46$ & $48.33 \pm 0.80$ & $52.82 \pm 3.20$ & $40.76 \pm 0.28$ & $40.38 \pm 2.98$ & $67.30 \pm 1.45$ & $43.33 \pm 3.48$ & $48.07 \pm 1.24$ & $41.66 \pm 2.31$ \\
\hline 4.5 & $25.51 \pm 1.90$ & $50.12 \pm 2.30$ & $59.23 \pm 1.20$ & $49.74 \pm 0.42$ & $48.46 \pm 1.16$ & $74.10 \pm 2.22$ & $50.12 \pm 4.22$ & $51.02 \pm 2.82$ & $47.17 \pm 2.98$ \\
\hline 5.0 & $25.89 \pm 1.20$ & $52.43 \pm 4.50$ & $62.94 \pm 0.42$ & $50.25 \pm 3.22$ & $51.92 \pm 0.94$ & $77.17 \pm 4.22$ & $51.66 \pm 3.60$ & $51.28 \pm 3.24$ & $51.53 \pm 0.88$ \\
\hline 5.5 & $33.46 \pm 3.30$ & $55.76 \pm 3.50$ & $66.02 \pm 0.88$ & $53.20 \pm 1.12$ & $53.20 \pm 2.78$ & $81.41 \pm 0.65$ & $56.79 \pm 1.26$ & $55.78 \pm 2.42$ & $55.76 \pm 4.22$ \\
\hline 6.0 & $34.35 \pm 2.41$ & $61.66 \pm 2.00$ & $73.33 \pm 1.14$ & $56.15 \pm 0.92$ & $58.07 \pm 0.52$ & $83.07 \pm 0.66$ & $58.84 \pm 3.25$ & $58.71 \pm 2.88$ & $58.33 \pm 3.36$ \\
\hline 6.5 & $35.51 \pm 1.46$ & $66.02 \pm 2.42$ & $77.30 \pm 0.82$ & $60.51 \pm 1.82$ & $62.69 \pm 0.68$ & $83.54 \pm 0.82$ & $64.10 \pm 1.67$ & $63.07 \pm 0.82$ & $59.23 \pm 0.12$ \\
\hline 7.0 & $38.07 \pm 1.60$ & $69.10 \pm 1.35$ & $83.46 \pm 0.84$ & $62.94 \pm 2.32$ & $65.51 \pm 3.22$ & $89.87 \pm 1.40$ & $65.89 \pm 3.20$ & $64.23 \pm 2.33$ & $60.38 \pm 2.98$ \\
\hline 7.5 & $38.84 \pm 0.88$ & $75.89 \pm 1.62$ & $83.97 \pm 1.32$ & $68.84 \pm 1.66$ & $70.38 \pm 1.12$ & $90.64 \pm 2.14$ & $69.10 \pm 1.20$ & $70.12 \pm 2.31$ & $61.15 \pm 0.28$ \\
\hline 8.0 & $38.97 \pm 1.22$ & $77.05 \pm 1.12$ & $84.35 \pm 1.42$ & $72.05 \pm 1.48$ & $76.79 \pm 1.92$ & $92.56 \pm 1.24$ & $75.25 \pm 2.64$ & $78.46 \pm 2.98$ & $61.80 \pm 0.18$ \\
\hline
\end{tabular}

The greater effectiveness of hydrogel formulation TF8 is due to rapid release of the drug (Table 3 ) in the tumor and exposure of the tumor cells to drug concentrations for a prolong period, thus causing more cell death than when release is slow.

\section{DISCUSSION}

We selected camptothecin as a model drug for this study, because its insolubility in water makes it difficult to administer systemically by other means and because of the potential applications of camptothecin and the insoluble camptothecin analogues in chemotherapy. Additionally, the pharmacologically important lactone ring of camptothecin and its analogs is unstable in the presence of human serum albumin which results in the conversion of the active drug to the inactive carboxylate form bound to albumin [1012]. This imposes a severe pharmacokinetic limitation on the systemic use of camptothecin and related compounds. An approach to overcoming this and other shortcomings of camptothecin and its analogs, especially their high systemic toxicity is to load it into a delivery system such as a chitosan based formulations which will protect the drug from hydrolysis and control its release over a prolonged period.

It is known that chitosan with block structures and lower degrees of deacetylation (DDA $=80$ $\%)$ are more readily biodegraded due to the presence of blocks of glucosamine moieties containing acetyl groups that serve as a substrate for lysozyme [13,14]. In this study estimation of \% tumor growth inhibition of CPT or chitosan/ $\beta-G P / \beta-C D / C P T$ was based on changes in tumor weight following hydrogel formulation. Several published studies describe the effect of formulation of chitosan/GP on the histology of the surrounding tissue. The effect of formulation in normal tissue has been described by Molinaro et al [15] as a mild non- specific inflammatory reaction.

In the present study the hydrogel was injected into the tumor. A report of the effect of chitosan/ $\beta-G P / \beta-C D / C P T$ injected into the MCF-7 tumor described the histology of the injected tumors as showing some degree of necrosis interspersed between viable tumor tissues with necrosis generally decreasing away from the center of the tumor. This pattern was seen for both CPT injected tumors and for those injected with chitosan/ $\beta-G P / \beta-C D / C P T$. In the case of the MCF-7 tumor CPT without excipients appears to have less tumoricidal effect. Chitosan/ $\beta-G P / \beta-$ $\mathrm{CD} / \mathrm{CPT}$ has been shown to Maximum tumoricidal activity in MCF-7 due to maximum release of drug. The effectiveness of the polymer hydrogel in delaying tumor growth clearly demonstrates the importance of this delivery system in maintaining an inhibitory level of drug over a long period of time. The main advantages of the biodegradable polymer implant such as chitosan/ $\beta-G P / \beta-C D$ used for the delivery of camptothecin to the mouse tumor are the high intra-tumoral concentrations of drug attainable, low systemic toxicity and the extended period of 
time over which the drug can be released in the tumor. The dose of camptothecin delivered using the hydrogel was $10 \mu \mathrm{g} / \mathrm{ml}$, which is 3 times the mean dose for MCF-7 cell. For the hydrogel, the delayed release of the drug and localization in the tumor prevents toxic systemic levels being reached.

\section{CONCLUSION}

This study demonstrates the effectiveness of using the biodegradable chitosan polymer to deliver high doses of camptothecin locally to a mouse tumor model. Growth of tumors treated in this fashion was retarded for significantly longer periods than were tumors treated with systemically administered camptothecin. Camptothecin delivered by intra-tumoral hydrogel showed no toxicity and was stable. Chitosan/ $\beta$ GP/ $\beta-C D$ hydrogel is a safe, effective, homogeneous, injectable and stable system for the delivery of camptothecin and this approach represents an attractive technology platform for the delivery of other clinically important hydrophobic drugs. The mechanism of gelation, which does not involve covalent cross-linkers, organic solvent or detergents, combined with a controllable residence time, renders this injectable biomaterial uniquely compatible with sensitive chemotherapeutic agents.

\section{ACKNOWLEDGEMENT}

The authors thank the Pranveer Singh Institute of Technology (PSIT), Kanpur, India, for assistance of the work, and also the TATA Memorial Center, Advanced Center for Treatment Research \& Education in Cancer (ACTREC), Mumbai, India, for providing the in vitro cell line studied of formulations to support for the successful completion of the work.

\section{REFERENCES}

1. Venditto VJ, Simanek EE. Cancer therapies utilizing the camptothecins: a review of the in vivo literature. Mol Pharm 2010; 7: 307-349.

2. Mi Z, Burke TG. Differential interactions of camptothecin lactone and carboxylate forms with human blood components. Biochemistry. 1994; 33: 10325-10336.
3. Gottlieb JA, Guarino AM, Call JB, Oliverio VT, Block JB. Preliminary pharmacologic and clinical evaluation of camptothecin sodium (NSC-100880). Cancer Chemother 1970; 54: 461-470.

4. Muggia FM, Creaven PJ, Hansen HH, Cohen $M H$, Selawry OS. Phase- I clinical trial of weekly and daily treatment with camptothecin (NSC-100880): correlation with preclinical studies. Cancer Chemother 1972; 56: 515-521.

5. Moertel CG, Schutt AJ, Reitemeier RJ, Hahn RG. Phase II study of Camptothecin (NSC-100880) in the treatment of advanced gastrointestinal cancer. Cancer Chemother 1972; 56: 95-101.

6. Thompson DO. Cyclodextrins enabling excipients: their present and future use in pharmaceuticals. Crit Rev Ther Drug Carrier Syst 1997; 14: 1-104.

7. Loftsson T, Brewster ME. Pharmaceutical applications of cyclodextrins, I: drug solubilization and stabilization. J. Pharm. Sci. 1996; 85: 1017-1025.

8. Challa R, Alka A, Javed A, Khar RK. Cyclodextrins in drug delivery: an updated review, AAPS Pharm. Sci. Tech. 2005; 6: E329-E357.

9. Jarry $C$, Chaput $C$, Chenite A, Renaud MA, Buschmann $M$, Leroux JC. Effects of steam sterilization on thermogelling chitosan-basedgels. J Biomed Mater Res. 2001; 58:127-135.

10. Fassberg J, Stella VJ. A kinetic andmechanistic study of the hydrolysis of camptothecin and some analogues. J. Pharm. Sci. 1992; 81: 676-684.

11. Hertzberg RP, Caranfa MJ, Holden KG, Jakas $D R$, Gallagher G, Mattern MR, Mong SM, Bartus JO, Johnson RK, Kingsbury WD. Modification of the hydroxy lactone ring of camptothecin: inhibition of mammalian topoisomerase I andbiologica I activity. J Med Chem. 1989; 32:715-720.

12. Burke TG, Mi Z. The structural basis of camptothecin interactions with human serum albumin: impact on drug stability. J Med Chem 1994; 37: 40-46.

13. Aiba S. Studies on chitosan: 4 Lysozymic hydrolysis of partially $\mathrm{N}$-acetylatedchitosans. Int $\mathrm{J}$ Biol Macromol 1992; 14: 225-228.

14. Tomihata K, Ikada $Y$. In vitro and in vivo degradation of films of chitin and its deacetylated derivatives. Biomaterials 1997; 18: 567-575.

15. Molinaro G, Leroux JC, Damas J, Adam A. Biocompatibility of thermosensitive chitosan-based hydrogels: an in vivo experimental approach to injectable biomaterials. Biomaterials 2002; 23: 27172722. 